\title{
Oxidation Kinetics of Thin and Ultrathin Fe Films
}

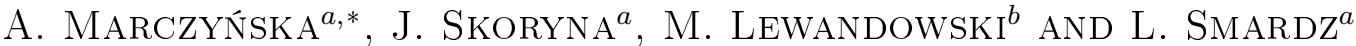 \\ ${ }^{a}$ Institute of Molecular Physics, Polish Academy of Sciences, M. Smoluchowskiego 17, 60-179 Poznań, Poland \\ ${ }^{b}$ NanoBioMedical Centre, Adam Mickiewicz University, Umultowska 85, 61-614 Poznań, Poland \\ We have studied oxidation kinetics of Fe thin films under atmospheric conditions using the fact that metallic \\ iron is a ferromagnet but ultrathin natural iron oxides are practically nonmagnetic at room temperature. As a \\ consequence, oxidation is associated with a loss in ferromagnetism. Fe thin films were deposited onto $1.5 \mathrm{~nm} \mathrm{~V}$ thick \\ buffer layer using UHV magnetron sputtering. As a substrate we have used $\mathrm{Si}(100)$ wafers with an oxidised surface. \\ Results show that all samples with an initial Fe thickness greater than $6 \mathrm{~nm}$ oxidize practically instantaneously, \\ whereby a constant amount of $2.5 \mathrm{~nm}$ of metal is transformed into oxides. For iron thickness lower than $6 \mathrm{~nm}$ \\ the time constant for oxidation increases considerably reaching a value of 30 days for the initial Fe thickness equal \\ to $4 \mathrm{~nm}$.
}

DOI: 10.12693/APhysPolA.127.549

PACS: 75.70.-i, 68.55.-a

\section{Introduction}

Binary iron oxides range from reasonable conductors (magnetite) to insulators and from ferrimagnets to antiferromagnets $[1,2]$. Wüstite $(\mathrm{FeO})$, magnetite $\left(\mathrm{Fe}_{3} \mathrm{O}_{4}\right)$, maghemite $\left(\gamma-\mathrm{Fe}_{2} \mathrm{O}_{3}\right)$, and hematite $\left(\alpha-\mathrm{Fe}_{2} \mathrm{O}_{3}\right)$ are the most common binary iron oxides. $\mathrm{FeO}$, the only $\mathrm{Fe}^{2+}$ binary oxide, has the cubic $\mathrm{NaCl}$ structure, and is an antiferromagnet below $200 \mathrm{~K}$. Magnetite, the oldest magnetic material known, is a mixed valence $\mathrm{Fe}^{2+} / \mathrm{Fe}^{3+}$ iron oxide with an inverse spinel structure, which is predicted to be a half-metal. The maghemite and hematite allotropes only contain $\mathrm{Fe}^{3+}$ ions. Maghemite presents the same inverse spinel-related structure as magnetite, albeit with some additional vacancies at the octahedral sites, and is insulating and ferrimagnetic. Hematite, the most stable phase in ambient conditions, is a rhombohedral iron oxide with the corundum structure, and a weak ferromagnet due to canted antiferromagnetism.

As is well known, all metallic thin films oxidize more or less under atmospheric conditions. Normally this process is depth limited such that an oxide covering layer with a well-defined thickness is formed by which the underlying metal is prevented from further oxidation. In this way one can obtain a self-stabilized bilayer structure [3]. It was reported in Ref. [3] that the polycrystalline and smooth cobalt films with an initial thickness of $d_{\mathrm{i}}>5 \mathrm{~nm}$ oxidize practically instantaneously, whereby a constant amount of $2.5 \mathrm{~nm}$ of metal is transformed into oxide. For $d_{\mathrm{i}}<5 \mathrm{~nm}$ the time constant for oxidation increases considerably and follows an approximately linear dependence with decreasing film thickness, reaching an extrapolated value of $\tau=190$ days for $d_{\mathrm{i}} \rightarrow 0$. This increasing time constant allows all samples with $d_{\mathrm{i}}<2.5 \mathrm{~nm}$ appear ferromagnetic for a long time due to a nonoxidized metallic rest.

${ }^{*}$ corresponding author; e-mail: agnmar@ifmpan.poznan.pl

For the iron films investigated here this bilayer system consists of two magnetically active materials, namely nonoxidised metallic iron covered by natural oxide layer. The oxidized ultrathin surface layer is predominantly consisted of mixture of $\alpha-\mathrm{Fe}_{2} \mathrm{O}_{3}$ and $\mathrm{FeO}$. Such a layer practically gives no ferromagnetic signal at room temperature. Metallic iron, on the other hand, is a well-known ferromagnet. Formation of oxide is at the expense of the metallic ferromagnet and therefore associated with a loss of the observed saturation magnetic moment. Using a sensitive vibrating sample magnetometer (VSM) we have measured the hysteresis curves of such iron films on an absolute scale and could evaluate a quantitative measure for the amount of ferromagnetic metal being transformed into practically nonmagnetic oxide.

\section{Experimental procedure}

The wedged samples with constant-thickness $\mathrm{V}$ and step-like wedged (areas with constant-thickness) Fe sublayers were prepared at room temperature using UHV $\left(5 \times 10^{-10}\right.$ mbar) magnetron sputtering [2-5]. As a substrate we have used $\mathrm{Si}(100)$ wafers with an oxidised surface to prevent a silicide formation $[3,6]$. Therefore we have applied a special heat treatment in UHV before deposition in order to obtain an epitaxial $\mathrm{SiO}_{2}$ surface layer $[3,6]$. The Fe-layers $\left(0.2 \leq d_{\mathrm{Fe}} \leq 20 \mathrm{~nm}\right)$ were deposited using a DC source. For preparation of constant thickness V-layers $\left(d_{\mathrm{v}}=1.5 \mathrm{~nm}\right)$ a RF source was used. To increase the signal in ex situ structural characterisations we have also prepared $\mathrm{V} / \mathrm{Fe}$ multilayers (MLs). The number of repetition of the base period was equal to 25 . The chemical composition and the cleanness of all layers was checked in situ, immediately after deposition, transferring the samples to an UHV $\left(4 \times 10^{-11} \mathrm{mbar}\right)$ analysis chamber equipped with X-ray photoelectron spectroscopy (XPS) $[4,5,7]$.

The morphology and roughness of the oxide layers were studied ex situ by atomic force microscopy (AFM). The structure of the V/Fe MLs was examined by standard $\theta-2 \theta$ X-ray diffraction with $\mathrm{Cu} K_{\alpha}$ radiation. 
The modulation wavelength was determined from the spacing between satellite peaks in the high and low-angle $\mathrm{X}$-ray diffraction patterns. The thicknesses of individual Fe and $\mathrm{V}$ sublayers were also determined using Xray fluorescence analysis (XRF) and X-ray reflectivity (XRR). The magnetic characterisation of the samples with constant-thickness sublayers was carried out using a vibrating sample magnetometer (VSM) at room temperature. Before measurements the step-like wedged $\mathrm{V} / \mathrm{Fe}$ bilayer was cut into pieces $(4 \times 15 \mathrm{~mm})$ with constant thickness sublayers. The total magnetic moments (M) were determined from the in-plane hysteresis loop measurements in magnetic fields up to $2 \mathrm{~T}$.

\section{Results and discussion}

In Fig. 1a and $\mathrm{b}$ we show AFM images measured for the $2 \mathrm{~nm} \mathrm{Fe} / 1.5 \mathrm{~nm} \mathrm{~V}$ and $16 \mathrm{~nm} \mathrm{Fe} / 1.5 \mathrm{~nm} \mathrm{~V}$ bilayers, respectively. The images show actual morphology of the oxidised Fe top layer. The roughness measured for the 2 and $16 \mathrm{~nm}$ iron top sublayers was about $0.2 \mathrm{~nm}$ and $0.3 \mathrm{~nm}$, respectively. The relatively low values of the roughness parameters revealed planar growth of the samples.
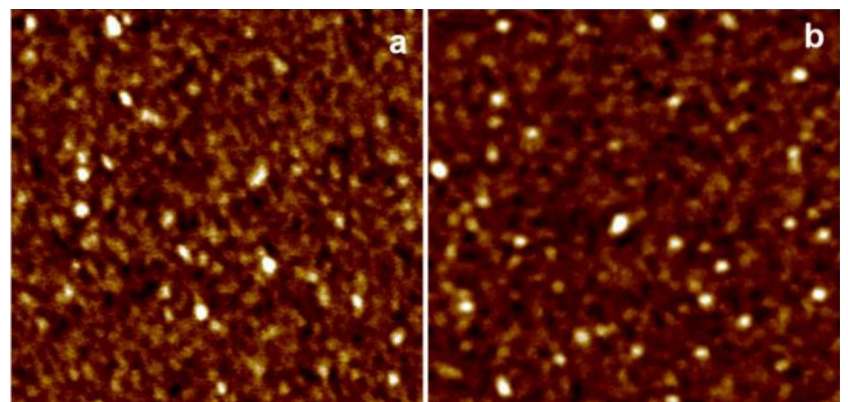

Fig. 1. AFM images $(500 \mathrm{~nm} \times 500 \mathrm{~nm})$ measured for the $2 \mathrm{~nm} \mathrm{Fe} / 1.5 \mathrm{~nm} \mathrm{~V}$ (a) and $16 \mathrm{~nm} \mathrm{Fe} / 1.5 \mathrm{~nm} \mathrm{~V}$ (b) bilayers.

The composition modulation of the $\mathrm{V} / \mathrm{Fe}$ MLs was confirmed in the high- and low-angle X-ray diffraction measurements by intense satellite peaks. The wavelengths of modulation calculated from these peaks were in agreement with those values determined from XRF. In the high-angle XRD patterns we have obseved central Bragg peak located between positions expected for reflections of bcc-Fe(110) and bcc-V(110) and at least two satellites for the thinner sublayers. The fitted roughness from the XRD data was about $0.3-0.4 \mathrm{~nm}$, which is in agreement with AFM measurements. Results on structural characterisation will be published in detail in a separate paper [8].

In Fig. 2 we show XPS spectrum of $1 \mathrm{~nm}$ Fe overlayer deposited on $1.5 \mathrm{~nm} \mathrm{~V}$ underlayer. As described above, practically no XPS signal is observed from potential contamination atoms like $\mathrm{O} 1 s$ and $\mathrm{C} 1 s$ (see Fig. 2). Furthermore, due to small roughness we have also observed no XPS signal from the $\mathrm{SiO}_{2} / \mathrm{Si}$ substrate.

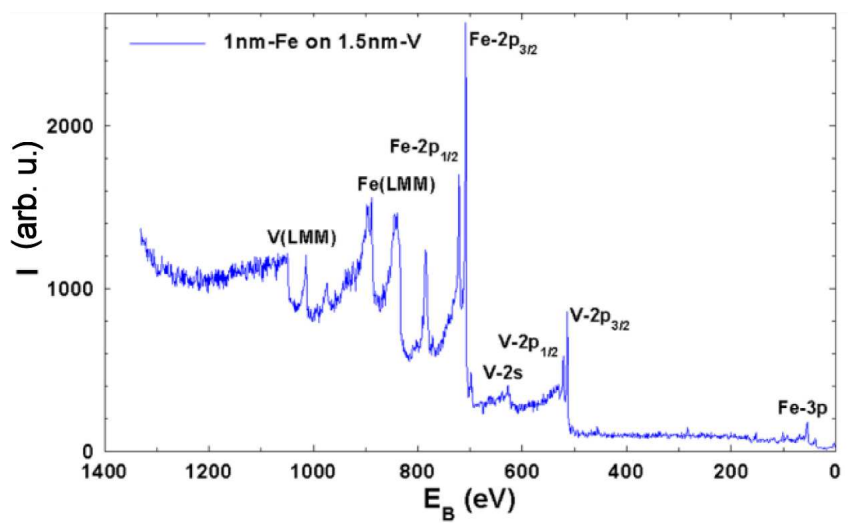

Fig. 2. XPS $\left(\mathrm{Al} K_{\alpha}\right)$ spectrum of the freshly prepared $1.5 \mathrm{~nm} \mathrm{V/1} \mathrm{nm} \mathrm{Fe} \mathrm{bilayer.}$

In the XPS experiment we have also studied the $\mathrm{Fe}$ layer growth on a $1.5 \mathrm{~nm} \mathrm{~V}$ underlayer. The freshly deposited $1.5 \mathrm{~nm} \mathrm{~V} / d_{0}$ Fe bilayer was in situ transferred from the preparation chamber to the analysis chamber, where the XPS Fe $2 p_{3 / 2}$ and $\mathrm{V} 2 p_{3 / 2}$ core level spectra were immediately measured in vacuum of $8 \times 10^{-11}$ mbar. Then the bilayer was transferred back to the preparation chamber and the deposition process of the Fe overlayer was continued. The above procedure (overlayer deposition and XPS core level measurements) was repeated until the $\mathrm{Fe} 2 p_{3 / 2}$ and $\mathrm{V} 2 p_{3 / 2}$ integral intensities were saturated. Practically no trace of oxygen (or any other contaminations) adsorption or surface oxide formation was detected during the transfer operation or XPS measurements $(\approx 10 \mathrm{~min})$.

From the exponential variation of the XPS Fe $2 p$ and $\mathrm{V} 2 p$ integral intensities with increasing overlayer Fe (V) thickness up to $5 \mathrm{~nm}$ we conclude that the $\mathrm{Fe}$ and $\mathrm{V}$ sublayers grow homogeneously in the planar mode. We have previously observed very similar growth mode for $\mathrm{Fe} / \mathrm{Ti}$ [5], $\mathrm{Fe} / \mathrm{Zr}$ [7], and $\mathrm{Co} / \mathrm{Ti}[9]$ bilayers.

Depending on the initial thickness $d_{\mathrm{i}}$ of the iron films, oxidation proceeds with a considerably different evolution in time. For samples with $d_{\mathrm{i}}>6 \mathrm{~nm}$ oxidation is accomplished within few days and is stopped after $2.5 \mathrm{~nm}$ of metal have been transformed into oxide. We have not observed any degradation of the saturation magnetization of those samples after several months. Apparently these samples have acquired a stable oxide top layer. However, for an initial Fe thickness of less than about $6 \mathrm{~nm}$ the same oxidation process is considerably prolonged and requires now weeks or months until its completion. In Fig. 3a we give an example of how this can be evidenced with magnetization measurements. Here two hysteresis curves are seen, taken on the same sample with an initial iron thickness of $6 \mathrm{~nm}$ after an exposure time of 1 and 52 days to ambient conditions. The more interesting observation is the decreasing saturation magnetization with time due to a continuing oxidation. With increasing oxide layer thickness (decreasing hysteresis signal) the hysteresis curve gets gradually broader, as can 


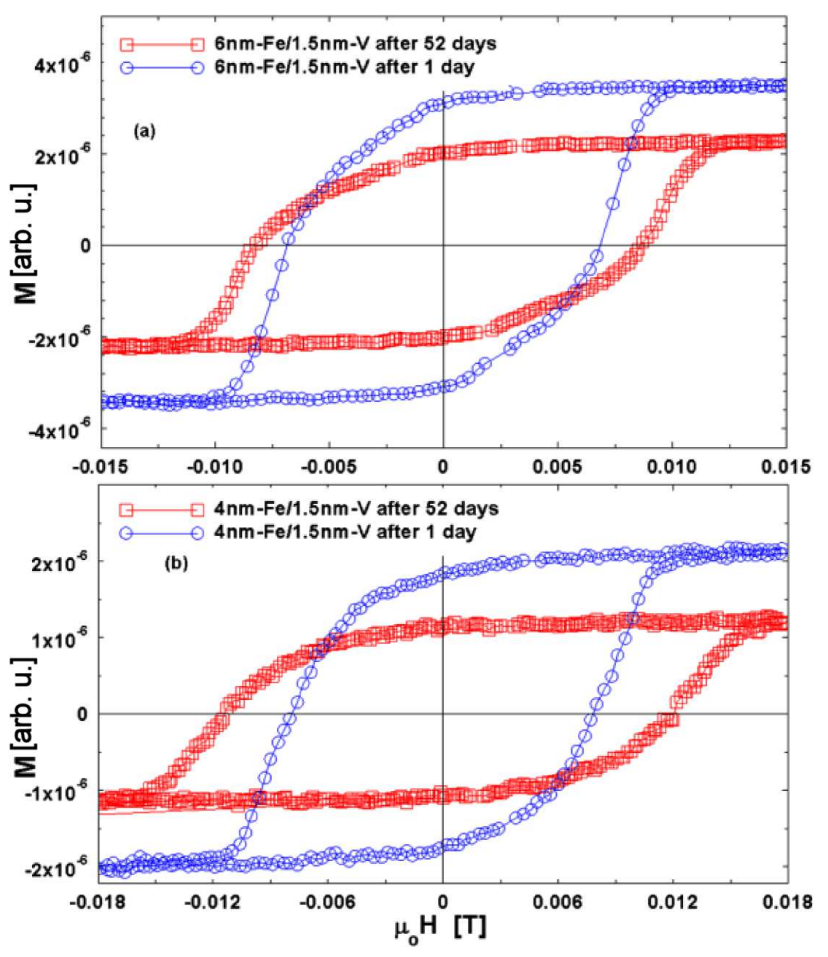

Fig. 3. In plane hysteresis loops of $6 \mathrm{~nm} \quad \mathrm{Fe} /$ $1.5 \mathrm{~nm} \mathrm{~V}$ (a) and $4 \mathrm{~nm} \mathrm{Fe} / 1.5 \mathrm{~nm} \mathrm{~V}$ (b) measured 1 day (circles) and 52 days (squares) after extraction from UHV conditions. Measurements were performed at room temperature.

best be seen in Fig. 3a. In Fig. 3b we give one further example for a still thinner sample with $d_{\mathrm{i}}=4 \mathrm{~nm}$. The XPS studies of the samples with $d_{\mathrm{i}}=4,6,10 \mathrm{~nm}$ after 100 days of oxidation showed that the surface layer is predominantly consisted of mixture of $\alpha-\mathrm{Fe}_{2} \mathrm{O}_{3}$ and $\mathrm{FeO}$. Assuming bulk magnetisation of the metallic iron rest it was possible to illustrate the time evolution of the oxidation process by giving the magnetically evaluated Fe thickness $d_{\mathrm{m}}$, as a function of time. Note the very different initial slopes of the curves. The sample with $d_{\mathrm{i}}=10 \mathrm{~nm}$ has reached its final thickness of $10-2.5=7.5 \mathrm{~nm}$ after a couple of days but the sample with $d_{\mathrm{i}}=4 \mathrm{~nm}$ is still oxidised within the time scale of this experiment. For a quantitative description of this process we apply a simple exponential law [3]: $d_{\mathrm{m}}(t)=d_{\mathrm{i}}-2.5\left(1-\mathrm{e}^{-t / \tau}\right)$. Equation contains only the time constant as a fit parameter and the solid and broken lines in Fig. 4 give the best fits which result in a satisfactory description of the experimental features. The time constant strongly depends on the initial Fe thickness and was estimated as 1, 25, and 30 days for $d_{\mathrm{i}}$ equal to 10,6 , and $4 \mathrm{~nm}$, respectively.

\section{Conclusions}

Results show that all samples with an initial Fe thickness greater than $6 \mathrm{~nm}$ oxidize practically instantaneously, whereby a constant amount of $2.5 \mathrm{~nm}$ of metal is transformed into oxides. For Fe thickness lower than

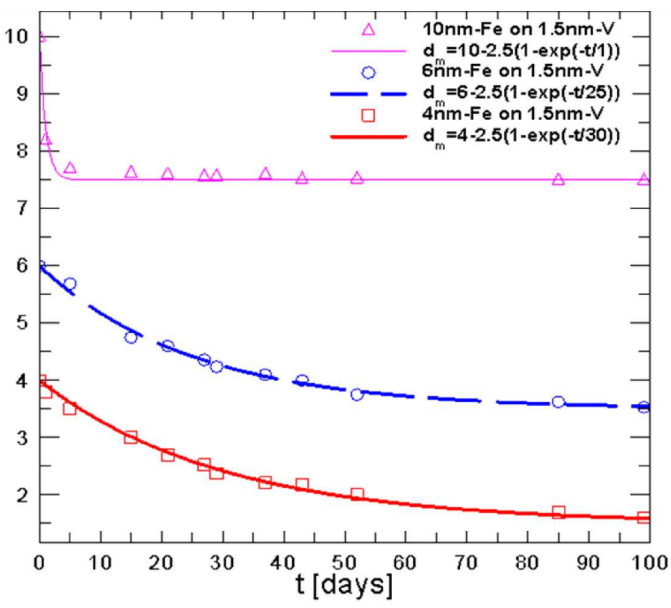

Fig. 4. Thickness of the ferromagnetic Fe layer $d_{\mathrm{m}}$, as a function of time. Solid and broken lines are exponentials using a thickness dependent time constant.

about $6 \mathrm{~nm}$ the time constant for oxidation increases considerably reaching a value of 30 days for the initial $\mathrm{Fe}$ thickness equal to $4 \mathrm{~nm}$.

\section{Acknowledgments}

This work was financially supported by the Polish Ministry of Science and Higher Education via "Iuventus Plus" programme (2012-2015; project number: IP2011 030071) and by the National Science Centre of Poland through "SONATA" programme ("MAGNETON" project; 2013-2016; project number: 2012/05/D/ST3/02855). M.L. would like to thank the Foundation for Polish Science for the START scholarship.

\section{References}

[1] R. Cornell, U. Schwertmann, The Iron Oxides, Wiley, New York 1997.

[2] M. Monti, B. Santos, A. Mascaraque, O. Rodriguez de la Fuente, M.A. Niño, T.O. Menteş, A. Locatelli, K.F. McCarty, J.F. Marco, J. de la Figuera, J. Phys. Chem. C 116, 11539 (2012).

[3] L. Smardz, U. Köbler, W. Zinn, J. Appl. Phys. 71, 5199 (1992).

[4] L. Smardz, M. Nowak, M. Jurczyk, Int. J. Hydrogen En. 37, 3659 (2012).

[5] K. Smardz, L. Smardz, Phys. Status Solidi B 243, 223 (2006).

[6] See for example, The Physics and Chemistry of $\mathrm{SiO}_{2}$ and the $\mathrm{Si}_{-} \mathrm{SiO}_{2}$ Interface, Eds. C.R. Helms, B.E. Deal, Plenum, New York 1988.

[7] L. Smardz, K. Smardz, Mater. Sci.-Poland 24, 821 (2006).

[8] A. Marczyńska, B. Szymański, J. Skoryna, L. Smardz, Acta Phys. Pol. A 127, 552 (2015).

[9] L. Smardz, Solid State Commun. 112, 693 (1999). 